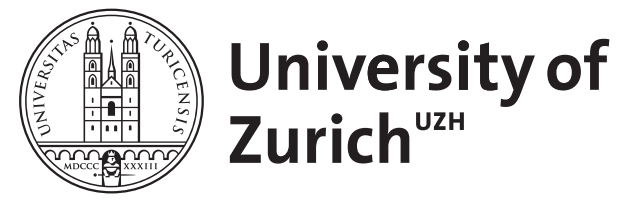

In vivo-Bestimmung der Knochenstruktur mittels 3D-Computertomographie

Laib, A ; Rüegsegger, P

DOI: https://doi.org/10.1515/bmte.1996.41.s1.41

Posted at the Zurich Open Repository and Archive, University of Zurich ZORA URL: https://doi.org/10.5167/uzh-154037

Journal Article

Published Version

Originally published at:

Laib, A; Rüegsegger, P (1996). In vivo-Bestimmung der Knochenstruktur mittels 3D-Computertomographie. Biomedizinische Technik. Biomedical engineering, 41(s1):41.

DOI: https://doi.org/10.1515/bmte.1996.41.s1.41 


\title{
In vivo-Bestimmung der Knochenstruktur mittels 3D-Computertomographie
}

\author{
Laib A., Rüegsegger P. \\ Institut für Biomedizinische Technik und Medizinische Informatik \\ Universität und ETH Zürich, CH-8044 Zürich, Schweiz
}

\section{EINLEITUNG:}

Osteoporose stellt mit wachsender Lebenserwartung der Bevölkerung ein immer grösseres Problem dar, das die betroffenen Individuen bei allfälligen Knochenbrüchen stark beeinträchtigen, in ungünstigen Fällen sogar immobilisieren kann. Das Frakturrisiko bei Osteoporosegefährdeten Patienten wurde bis jetzt aufgrund von Knochendichtemessungen bestimmt. Die Knochendichte ist aber nur einer der Faktoren, welcher die Knochenstärke bestimmt. Ebenso wichtig kann die Struktur des Knochens sein. Um die komplexe 3D-Architektur von spongiösem Knochen in vivo zu bestimmen, wird mit einem speziellen CT ein grösserer Knochenabschnitt hochauflösend aufgenommen. Aus diesen Daten wird die Knochenstruktur extrahiert und deren morphometrische Eigenschaften bestimmt.

\section{METHODE:}

An unserem Institut wurde ein spezieller ComputerTomograph entwickelt und gebaut, der Aufnahmen der menschlichen Extremitäten mit einer isotropen (nominellen) Auflösung von $165 \mu \mathrm{m}$ ermöglicht. Das Messsystem ist so konzipiert, dass die Strahlenbelastung einer Untersuchung nicht grösser ist als diejenige einer normalen Röntgenuntersuchung, mit dem wesentlichen Unterschied jedoch, dass nur ein $1 \mathrm{~cm}$ breiter Streifen am Unterarm belastet wird. Dieser $1 \mathrm{~cm}$ breite Bereich wird mit 60 dünnen Tomogrammen überdeckt. Für die Messung wird eine Röntgenquelle mit Linienfokus und ein 2D-Detektorarray verwendet, welche die gleichzeitige Messung von 10 Schichtbildern erlaubt.

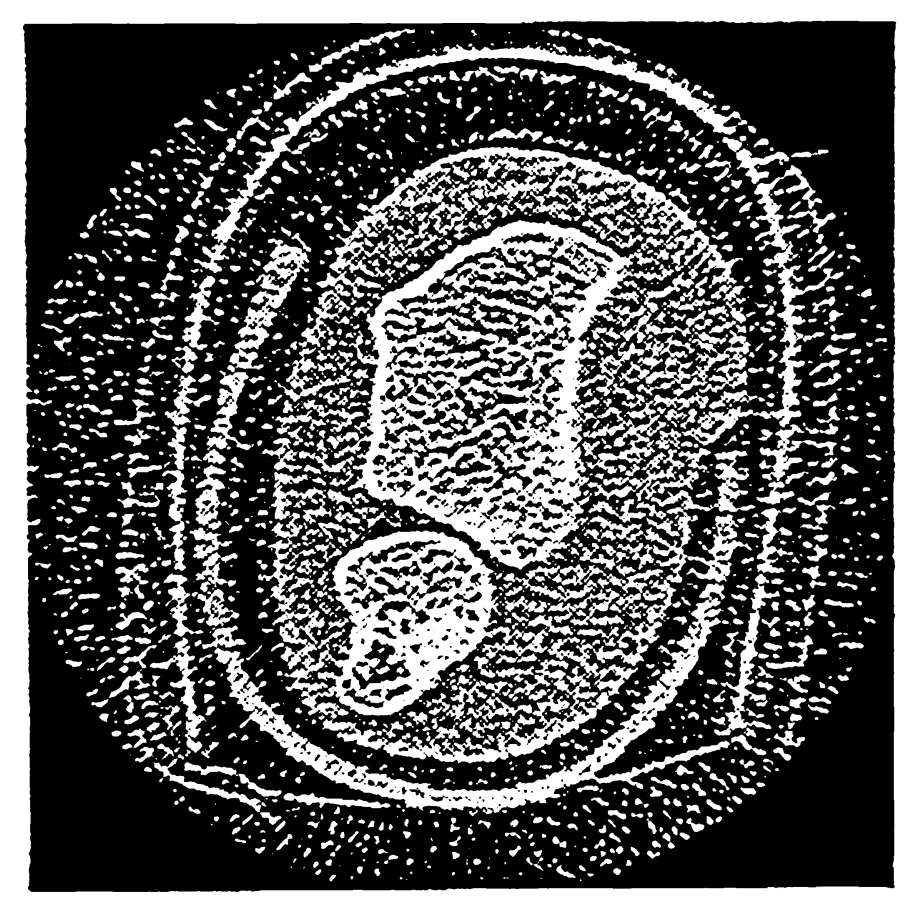

Figur 1: $\quad$ Eine Schicht einer CT Unterarm-Aufnahme. Der Unterarm ist zur Immobilisierung in eine Messmulde eingebettet.
Vom erhaltenen Datensatz wird das Gebiet des Radius extrahiert, anschliessend segmentiert, und das Knochenskelett der Spongiosa wird bestimmt und die Anzahl Trabekel pro Volumen daraus berechnet. Die Patientenmessungen werden in einem zeitlichen Abstand von 3 oder 6 Monaten am gleichen Messort wiederholt und die Änderung sowohl der Knochendichte als auch der Knochenskelettstruktur wird untersucht.

\section{ERGEBNISSE:}

Erste Ergebnisse zeigen eine gute Reproduzierbarkeit der Strukturparameter. Untersuchungen der Auflösungsabhängigkeit dieser Parameter zeigen aber auf, dass eine räumliche Auflösung von ca. $100 \mu \mathrm{m}$ nötig wäre, um die Breite der Knochentrabekel genau zu ermitteln. Diese Auflösung bedingt aber Messungen mit relativ hoher Strahlenbelastung. Als neue Methode bietet sich die Lokale Tomographie an, wo nur ein Teil des Objektes bestrahlt, aufgenommen und lokal rekonstruiert wird.
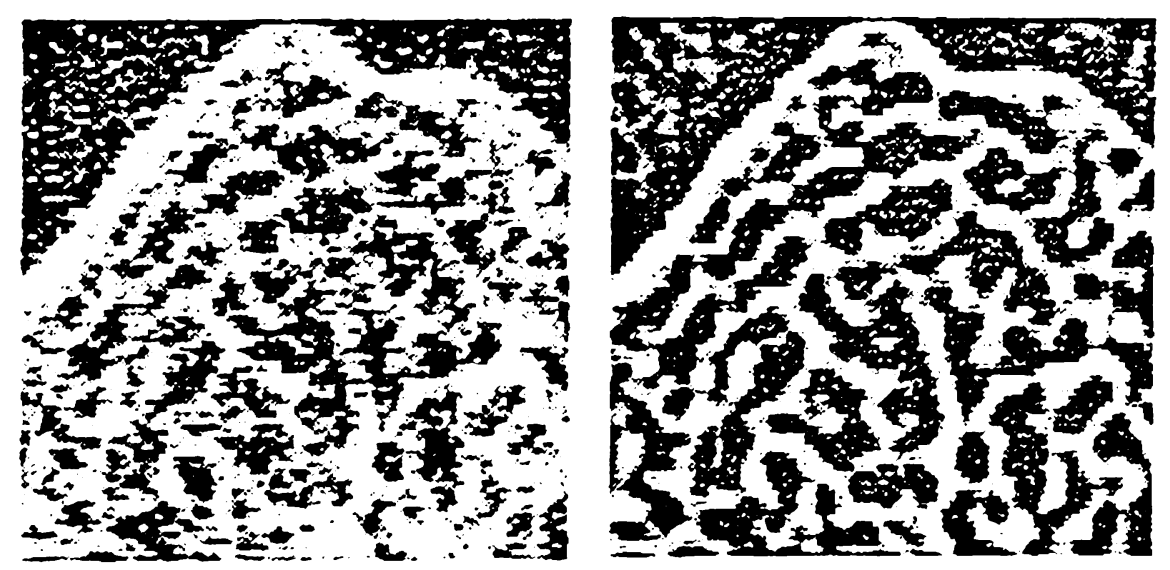

Figur 2: Ausschnitt einer globalen TomographieAufnahme eines explantierten Radius links, gleiches Gebiet mit Lokaler Tomographie rechts.

Die gelieferten Bilder enthalten keine quantitativen Dichteinformationen mehr, aber die gewünschte Strukturinformation ist vorhanden.

\section{DISKUSSION:}

Die Daten mit $165 \mu \mathrm{m}$ Auflösung zeigen vielversprechende Ergebnisse, insbesondere für die Parameter der trabekulären Anzahl und des trabekulären Abstands. Um zusätzlich die Dicke der einzelnen Trabekel akkurat zu bestimmen, kann ein kleineres Knochengebiet hochauflösend mit Lokaler Tomographie dosisschonend aufgenommen werden. Zusammen mit den Daten einer niedriger auflösenden, globalen Aufnahme können sowohl die Knochendichte als auch die morphometrischen Parameter berechnet und daraus die Knochenstärke bestimmt werden. 